\title{
Saw torque transducers for disturbance rejection and tracking control of multi-inertia servo-drive systems
}

\author{
${ }^{\perp}$ T. M. O'Sullivan, N. Schofield, C. M. Bingham \\ Electrical Machines and Drives Research Group \\ Dept. of Electronic and Electrical Engineering, University of Sheffield \\ SHEFFIELD, SI 3JD, UK. Tel. +44 (0) 1142225195 \\ ${ }^{\perp}$ Corresponding author: t.osulłivan@sheffield.ac.uk
}

\begin{abstract}
The paper proposes a resonance ratio control (RRC) technique for the coordinated motion control of multiinertia mechanical systems, based on the measurement of shaft torque via a SAW-based torque sensor. Furthermore, a new controller structure, RRC plus disturbance feedback is proposed, which enables the controller to be designed to independently satisfy tracking and regulation performance. A tuning method for the RRC structure is given based on the ITAE index, normalized as a function of the mechanical parameters enabling a direct performance comparison between a basic proportional and integral (PI) controller. The use of a reduced-order state observer is presented to provide a dynamic estimate of the load-side disturbance torque for a multi-inertia mechanical system, with an appraisal of the composite closedloop dynamics. It is shown that the integrated formulation of the tuning criteria enables lower bandwidth observers to be implemented with a corresponding reduction in noise and computational load. The control structures are experimentally validated via a purpose designed test facility and demonstrate significant improvement in dynamic tracking performance, whilst additionally rejecting periodic load side disturbances, a feature previously unrealisable except by other, high-gain control schemes that impose small stability margins.
\end{abstract}

\section{INTRODUCTION}

With servo drive systems continually improving in performance capability, improved control of the electromechanical system dynamics is becoming an increasingly common industrial requirement. However, impulsive transient demands from such systems can excite mechanical torsional resonances in the associated drivetrain, ultimately leading to controller instability. Practical mechanical drive systems can be complex, incorporating several non-stiff interconnecting shafts and elastic couplings. The dominant fundamental resonant frequency, however, is typically between $<300 \mathrm{~Hz}$, which often overlaps with the closed-loop dynamic bandwidth imposed by the control scheme. The higher resonant modes often remain relatively unexcited, allowing a large proportion of typical industrial drive systems to be accurately modelled using a two-inertia approximation.

Until recently, difficulties in acquiring reliable, low-noise, low-cost, shaft torque transducers that are non-invasive to the mechanical drive system, have precluded the use of direct torque feedback in all but a minority of specialised closed-loop servo-drive systems. Often, commonly employed torque transducers viz. strain gauge, optical and inductive devices, are too mechanically compliant when incorporated in a drive system, thereby degrading stability margins and reducing closed-loop bandwidth. Moreover, the additional cost associated with their integration is prohibitive.

Here then, an investigation in applying a new, low-cost, non-contact torque measurement device, based on surface acoustic wave (SAW) technology [1], is reported for use in high performance brushless machine-based servo applications. It will be shown that direct measurement of shaft torque, coupled with a resonance ratio control strategy [2-4], can significantly enhance servo-drive system dynamics. Additionally, the use of observers is presented to directly incorporate shaft-torque feedback to provide loadside torque disturbance rejection, and the ability to design controllers for independently satisfying closed-loop tracking, and regulation performance. Conventionally the closed-loop dynamics induced by the presence of a state observer is often ignored since the observer is usually designed to be much faster than the system dynamics. However in practice, a fast observer may lead to the undesirable amplification of high frequency noise particularly if the feedback sensors are noisy (such a the speed output from a quantized encoder). This paper therefore, provides a tuning methodology that considers the additional dynamics induced by the observer thus enabling a further reduction in observer speed.

Moreover, since the SAW torque transducer exhibits a high sensitivity and bandwidth, and is largely unaffected by electromagnetic noise, it can be directly integrated into an electrical machine assembly without affecting the mechanical stiffness of the system.

\section{TWO-INERTIA MECHANICAL SYSTEM}

Fig. 1(a) shows the schematic of a mechanical system containing two lumped inertias $J_{m}$ and $J_{d}$ representing the motor and load, respectively. The inertias are coupled via a shaft of finite stiffness $K_{m d}$, which is subject to torsional torque $t_{m d}$, and excited by a combination of electromagnetic torque $t_{e}$, and load torque perturbations $t_{d}$. The motor velocity is designated $\omega_{m}(\mathrm{rad} / \mathrm{s})$ and load velocity $\omega_{t}(\mathrm{rad} / \mathrm{s})$. Since the damping losses are generally low, they are neglected without significantly affecting the accuracy of the forgoing analysis [2-8].

Fig. 1(b) shows a dynamic model of the two-inertia mechanical servo-drive system. The transfer functions from 
electromagnetic torque to motor speed, and electromagnetic torque to load speed, are described by:

$$
\frac{\omega_{m}(s)}{t_{e}(s)}=\frac{s^{2}+\omega_{a}^{2}}{J_{m} s^{3}+J_{m} \omega_{n}^{2}}
$$$$
\text { (1) } \quad \frac{\omega_{d}(s)}{t_{e}(s)}=\frac{\omega_{a}^{2}}{J_{m} s^{3}+J_{m} \omega_{n}^{2} s}
$$

where $\omega_{h}$ is the anti-resonant frequency, $\omega_{h}$ the resonant frequency, and $R$ the load-motor inertia ratio:

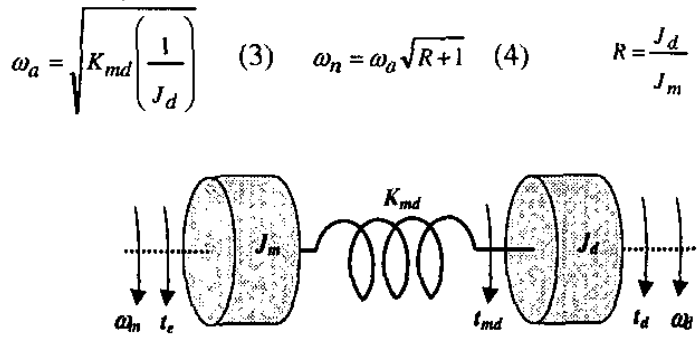

(a) Mechanical schematic

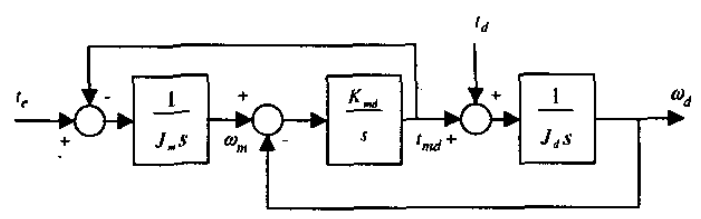

(b) Control block diagram

Figure 1. Two-inertia servo-drive system

\section{PI \& RRC CONTROL STRUCTURES}

\section{A. Tracking dynamic performance}

A classical PI-type control structure is illustrated in Fig. 2(a). This control structure can be extended by augmenting a feedback signal proportional to shaft torque as illustrated Fig. 2(b), the feedback transfer gain being $K_{s}$. Applying a feedback signal proportional to torsion in this manner is commonly referred to as Resonance Ratio Control (RRC) [2 4].

The resulting closed-loop transfer functions from reference input to load speed (tracking dynamics) for both PI and RRC structures are given in (6), with the equivalent inertia ratio, $\tilde{R}$ given in (7) for completeness. It is noted that $\tilde{R} \rightarrow R$ as $K_{s} \rightarrow 0$. Tracking performance of the two control structures are evaluated using the 'integral of time multiplied by absolute error' (ITAE) performance index for a step input, to penalise overshoot and settling time for a specified rise-time or equivalent $-3 \mathrm{~dB}$ bandwidth [9].

The step tracking dynamics of the PI-type scheme of Fig. 2(a), can be completely defined by the location of its closedloop poles. Equating the coefficients of the characteristic equation (the denominator of (6) when $K_{s}=0$ ), with the coefficients of the $4^{\text {th }}$-order ITAE polynomial (8), the system can be tuned for optimum performance, where $\omega_{x}$ is the equivalent $-3 \mathrm{~dB}$ bandwidth.

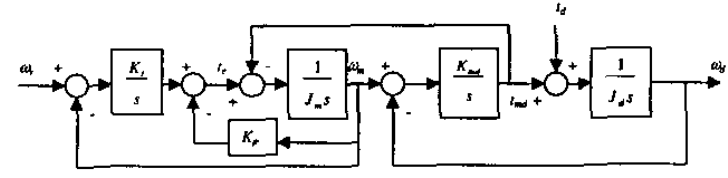

(a) PI-type controller

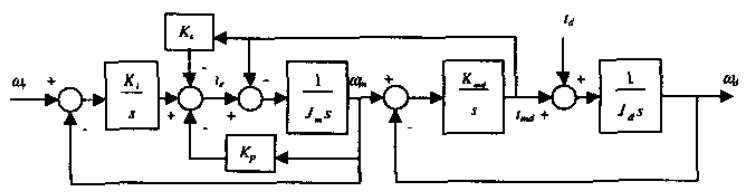

(b) RRC controller

Figure 2. Control structures for a two-inertia mechanical model of a servodrive system

$$
\begin{gathered}
\frac{\omega_{d}}{\omega_{r}}=\frac{K_{i} \omega_{a}^{2}}{J_{m} s^{4}+K_{p} s^{3}+\left(J_{m} \omega_{a}^{2}(1+\tilde{R})+K_{i}\right) s^{2}+K_{p} \omega_{a}^{2} s+K_{i} \omega_{a}^{2}} \\
\tilde{R}=R\left(1+K_{s}\right) \\
s^{4}+2.1 \omega_{x} s^{3}+3.4 \omega_{x}{ }^{2} s^{2}+2.7 \omega_{x}{ }^{3} s+\omega_{x}
\end{gathered}
$$

\begin{tabular}{|c|}
\hline TABLE I. \\
RRC CONTROLLER GAINS FOR \\
OPTIMAL LOAD SIDE \\
TRACKING PREFORMANCE. \\
\hline$K_{p} \approx 1.85 \omega_{a} J_{m}$ \\
\hline$K_{i} \approx 0.6 \omega_{a}{ }^{2} J_{m}$ \\
\hline$K_{s}=\frac{J_{m}}{J_{d}}-1$ \\
\hline
\end{tabular}

Moreover, equating terms gives the optimum $-3 \mathrm{~dB}$ tracking bandwidth as $\omega_{x}=0.88 \omega_{h}$, when the motor and load inertias are matched, i.e. when $R=1$. For many servo-drive system applications, where a gear reduction stage is employed, the reflected load inertia is reduced by $N^{2}$, where $N$ is the gear reduction ratio. By analysing the closed-loop pole restrictions, the load side tracking performance of the PI controller and two-inertia system shows an increasingly underdamped response as $R$ reduces from the optimal value, or as $\omega_{x}$ becomes greater than $\omega_{k}$. However, by using the RRC structure when $R \neq 1, R$ can be virtually adjusted to $\tilde{R}$ by the appropriate selection of $K_{s}$, thereby theoretically providing optimum performance for any $R$. Table I gives the optimal gains for the RRC controller.

\section{B. Regulation dynamic performance}

Equation (9) provides the closed-loop transfer function from disturbance torque to load speed (regulation dynamics), 
for both the PI $\left(K_{s}=0\right)$ and RRC controllers, where the inertia ratio $\tilde{R}$ for the RRC controller is expressed in (7).

From (9), it can be seen that the location of the closedloop zeros cannot be assigned independently of the closedloop poles, and, consequently, the closed-loop regulation and tracking dynamics cannot be independently tuned.

$$
\begin{aligned}
& \frac{\omega_{d}}{t_{d}}=\frac{s}{J_{d}} \frac{J_{m} s^{2}+K_{p} s+\left(K_{i}+K_{n d}\left(1+K_{s}\right)\right)}{J_{m} s^{4}+K_{p} s^{3}+\left(J_{m} \omega_{a}^{2}(1+\tilde{R})+K_{i}\right) s^{2}+K_{p} \omega_{a}^{2} s+K_{i} \omega_{a}^{2}} \\
& \frac{\omega_{d}}{t_{d}}=\frac{s}{J_{d}} \frac{J_{m} s^{2}+\left(K_{p}-K_{m d} K_{d d}\right) s+\left(K_{i}+K_{m d}\left(1+K_{s}-K_{p d}\right)\right)}{J_{p} s^{3}+\left(J_{m} \omega_{a}^{2}(1+\tilde{R})+K_{i}\right) s^{2}+K_{p} \omega_{a}^{2} s+K_{i} \omega_{a}^{2}}
\end{aligned}
$$

Here, an extended RRC control structure is introduced in Fig. 3(a), which includes an additional feedback signal proportional to the disturbance torque $K_{p d}$ and its derivative $K_{d d}$. Equation (10) now gives the modified closed-loop transfer function describing the regulation dynamics, from where it can be seen that the closed-loop poles and zeros can be independently assigned. Assigning the closed-loop zeros to the imaginary axis (i.e. no damping of the complex conjugate zeros) for a user-defined frequency, $\omega_{j}$, will reject a periodic load-side disturbance of the same frequency. Equations (11) and (12) gives the required disturbance torque gains.

$$
K_{p d}=\frac{K_{m d}\left(1+K_{s}\right)-\omega_{r j}^{2} J_{n z}+K_{i}}{K_{n d}} \quad \text { (11) } \quad K_{d d}=\frac{K_{p}}{K_{n d}} .
$$

Periodic load-side disturbances are a common feature of industrial automated production systems, for instance, where objects are dropped at equal time intervals onto a conveyer belt. For such systems, disturbance torque cannot be sensed directly and the extended control scheme shown in Fig. 3(a) therefore requires an observer to provide a dynamic estimate. Assuming the disturbance torque is a statevariable, and its derivative is zero, a state-variable representation of the system can be obtained that includes the disturbance torque as a state (12).

The measured output states can be used to reduce the complexity of the observer. In this case both $\omega_{m}$ and $t_{m d}$ are sensed, thereby reducing the required order of the observer to two. It therefore takes the dynamic structure shown in Fig. 3(b). Letting $\psi=\left[q_{1} q_{2}\right]^{\mathrm{t}}$ be an internal state vector of the observer, and $G=\left[g_{1} g_{2}\right]^{\mathrm{t}}$ be the observer gain vector, (13) is obtained from Gopinath's reduced order theorem [9], where $X_{1}$ and $X_{2}$ represent the measured and estimated state variables, respectively. The transfer function describing the relationship between the observed and actual load-torque can be obtained from (12) and is given by (14) where the denominator equation describes the observer pole locations. The observer poles are therefore assigned according to the coefficients of the $2^{\text {nd }}$ order ITAE polynomial given in (15), where $2 \zeta_{o p}=1.414$ and $\omega_{b b}$ is the equivalent $-3 \mathrm{~dB}$ observer bandwidth. The observer gains $G_{1}$ and $G_{2}$ are given by (16) and (17) respectively.

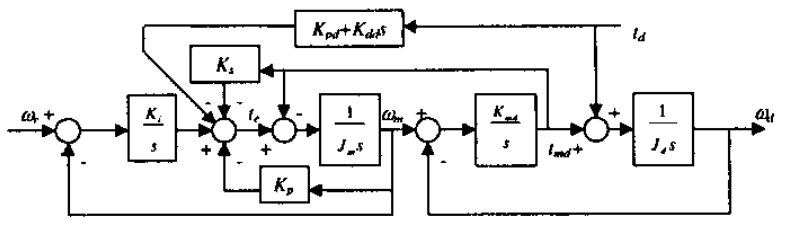

(a) RRC controller plus disturbance feedback

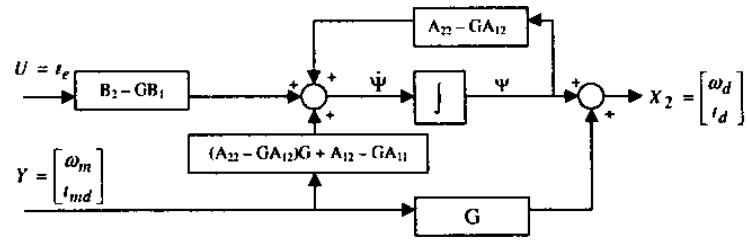

(b) Reduced order observer

Figure 3. Control structures for a two-inertia mechanical model of a servo drive system

$$
\begin{aligned}
& \frac{d}{d t}\left[\begin{array}{c}
\omega_{m} \\
t_{m d} \\
\hdashline \omega_{d} \\
t_{d}
\end{array}\right]=\left[\begin{array}{cc:cc}
0 & -\frac{1}{J_{m}} & 0 & 0 \\
K_{m d} & 0 & K_{m d} & 0 \\
\hdashline 0 & \frac{1}{J_{d}} & 0 & -\frac{1}{J_{d}} \\
0 & 0 & 0 & 0
\end{array}\right] \cdot\left[\begin{array}{c}
\omega_{m 1} \\
t_{m d} \\
\hdashline \omega_{d} \\
t_{d}
\end{array}\right]+\left[\begin{array}{c}
\frac{1}{J_{m}} \\
0 \\
\hdashline 0 \\
0
\end{array}\right] s_{e} \\
& =\left[\begin{array}{c:c}
A_{11} & A_{12} \\
\hdashline A_{21} & A_{22}
\end{array}\right] \cdot\left[\begin{array}{l}
X_{1} \\
\hdashline X_{2}
\end{array}\right]+\left[\begin{array}{c}
B_{1} \\
B_{2}
\end{array}\right] U
\end{aligned}
$$

$$
\begin{aligned}
& \dot{\psi}=X_{2}\left(A_{22}-G A_{12}\right)+X_{1}\left(A_{21}-G A_{11}\right)+\left(B_{2}-G B_{1}\right) u \\
& \psi=X_{2}-G X_{1} \\
& \frac{G_{2} \omega_{a}{ }^{2}}{s^{2}+s\left(-G_{1} K_{m d}\right)+G_{2} \omega_{a}^{2}} \quad \text { (14) } \quad s^{2}+2 \varsigma_{o b} \omega_{o b} s+\omega_{o b}{ }^{2} \\
& G_{1}=\frac{-1.4 \omega_{o b}}{K_{n d}} \quad \text { (16) } \quad G_{2}=\frac{\omega_{o b}{ }^{2}}{\omega_{a}^{2}}
\end{aligned}
$$

If the observer pole locations are assigned to be much greater than the closed loop zeros in (10), it can be assumed that the observer dynamics do not unduly influence the performance of the closed-loop regulation dynamics. However, in practice the observer poles cannot be placed significantly higher since there exists a trade-off between the bandwidth of the observer and filtering of high frequency noise generated by the sensing devices and/or the power amplifier. Moreover, in the case of the proposed control scheme, where the derivative of the observed load torque is required, the attenuation of high frequency noise is of greater importance if the control scheme is to be practically realisable. 
Consider Fig. 4, which is identical to the control structure of Fig. 3(a) except the observer dynamics are included in the disturbance feedback. Equation (18) now gives the modified closed-loop transfer function describing the regulation dynamics, where it can be seen that the numerator now contains two pairs of complex zeros that can be independently assigned by the selection of the observer gains $G_{l}$ and $G_{2}$, and the disturbance feedback gains $K_{p d}$ and $K_{d d}$. Thus, by proper adjustment of these gains, the closedloop system dynamics can be tuned to reject a specific user defined frequency whilst eliminating the effects of the observer dynamics on the rejection performance, i.e. a relatively slow disturbance observer can be implemented, attenuating high frequency noise, without sacrificing the control objective.

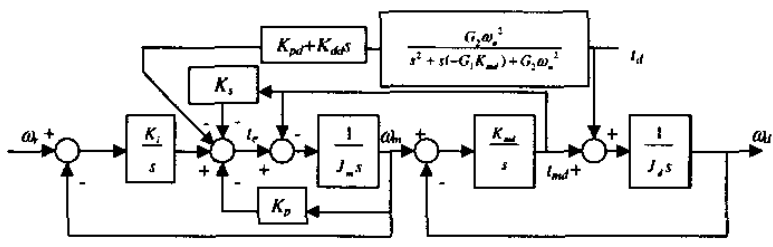

Figure 4. RRC controller plus observer disturbance feedback

$$
\begin{aligned}
& \frac{\omega_{d}}{t_{d}}= \\
& \frac{s}{J_{d}} \frac{\left\{\begin{array}{l}
J_{m} s^{4}+\left(K_{p}-G_{3} K_{m d} J_{m}\right) s^{3} \\
+\left(G_{4} \omega_{a}{ }^{2} J_{m}-G_{3} K_{m d} K_{p}+\left[K_{i}+K_{m d}\left(1+K_{s}\right)\right]\right) s^{2} \\
+\left(G_{4} \omega_{a}{ }^{2} K_{p}-G_{3} K_{m d}\left[K_{i}+K_{m d}\left(1+K_{s}\right)\right]-J_{d} K_{d d} G_{4} \omega_{a}{ }^{2}\right) s \\
+G_{4} \omega_{a}{ }^{2}\left[K_{i}+K_{m d}\left(1+K_{s}\right)\right]-J_{d} K_{p d} G_{4} \omega_{a}{ }^{4}
\end{array}\right\}}{\left\{\begin{array}{l}
\left.J_{m} s^{4}+K_{p} s^{3}+\left(J_{m} \omega_{a}{ }^{2}(1+\tilde{R})+K_{i}\right) s^{2}+K_{p} \omega_{a}{ }^{2} s+K_{i} \omega_{a}{ }^{2}\right) \\
\left(s^{2}-G_{3} K_{m d} s+G_{4} \omega_{a}{ }^{2}\right)
\end{array}\right\}}
\end{aligned}
$$

$$
\left(s^{2}+\omega_{r j}{ }^{2}\right)\left(s^{2}+2 \varsigma_{2} \omega_{2}+\omega_{2}^{2}\right)
$$

Equation (19) shows a general expression describing two pairs of complex zeros, where the first root represents the user defined rejection frequency, $\omega_{j j}$ (damping equals zero), and the other root defines the arbitrary location of the second pair of complex zeros. By comparing the numerator of (18) with (19), expressions can be derived that enable $\omega_{j}$ and the observer pole locations to be independently assigned as follows:

$$
\begin{gathered}
\zeta_{2} \omega_{2}=\frac{K_{p}+2 \zeta_{o b} \omega_{v b} J_{m b}}{2 J_{m}} \\
\omega_{2}=\sqrt{\frac{\omega_{o b}{ }^{2} J_{m}+K_{n d d}\left(1+K_{s}\right)+K_{i}+2 \zeta_{o b} \omega_{o b} K_{p}-\omega_{a}{ }^{2}}{J_{m}}} \\
K_{p d t}=\frac{G_{4} \omega_{o}{ }^{2}\left[K_{i}+K_{m d}\left(1+K_{s}\right)\right]-\omega_{r j}{ }^{2} \omega_{2}{ }^{2} J_{m}}{J_{d} G_{4} \omega_{a}{ }^{4}}
\end{gathered}
$$

$$
K_{d d}=\frac{G_{4} \omega_{a}^{2} K_{p}+2 \zeta_{2} \omega_{j} \omega_{2} J_{m}-G_{3} K_{m d}\left[K_{i}+K_{m d}\left(1+K_{s}\right)\right]}{J_{d} G_{4} \omega_{a}^{4}}
$$

\begin{tabular}{|l|l|}
\hline \multicolumn{2}{|c|}{ TABLE II. } \\
\multicolumn{2}{|c|}{ MECHANICAL PARAMETERS } \\
\hline$R$ & 0.5 \\
\hline$J_{m}$ & $0.0029 \mathrm{kgm}^{2}$ \\
\hline$J_{d}$ & $0.00145 \mathrm{kgm}^{2}$ \\
\hline$K_{m d}$ & $110 \mathrm{Nm} / \mathrm{rad}$ \\
\hline$\omega_{h}$ & $275.43 \mathrm{rad} / \mathrm{s}(34 \mathrm{~Hz})$ \\
\hline
\end{tabular}

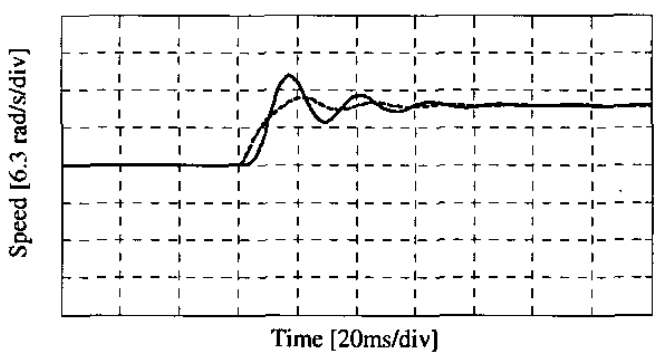

(a) PI controller with $\omega_{x}=0.88 \omega_{a}$

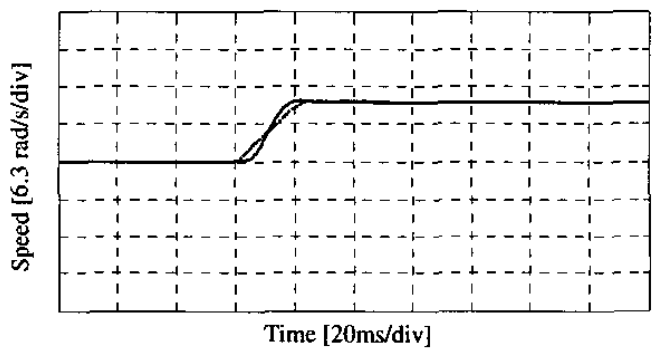

(b) RRC controller with $\omega_{x}=0.88 \omega_{n}$

Figure 5. Simulated tracking step responses for the two-inertia servo-drive system

\section{SIMULATED PERFORMANCE COMPARISON}

To verify the theoretical analysis, an experimental multimass test facility has been assembled as discussed in the next section. The mechanical parameters of the test facility are given in Table II. Using the presented control structures, dynamic simulation models of the experimental closed-loop system are now employed to show performance comparisons.

\section{A. Tracking performance}

The RRC controller gains $K_{p}, K_{i}$ and $K_{s}$ are tuned optimally according to Table I. Fig. 5 shows simulated results from the system in response to a step change in reference velocity of $10 \mathrm{rad} / \mathrm{s}$. Fig. $5(\mathrm{a})$ shows the dynamics resulting from the PI control structure, whilst Fig. 5(b) shows the RRC-induced dynamics. It can be seen that since 
$R<1(R=0.5)$, the PI controller imparts an underdamped response, particularly at the load side, whilst the RRC controller 'virtually' increases $R$ resulting in optimal loadside tracking performance.

\section{B. Regulation performance}

With identical control gains as for the tracking performance evaluation, the regulation performance is evaluated via the closed-loop systems ability to reject a sinusoidal load-side disturbance. By way of example, the load-side disturbance is a $4 \mathrm{Nm}, 62.8 \mathrm{rad} / \mathrm{s}(10 \mathrm{~Hz})$ sine wave.

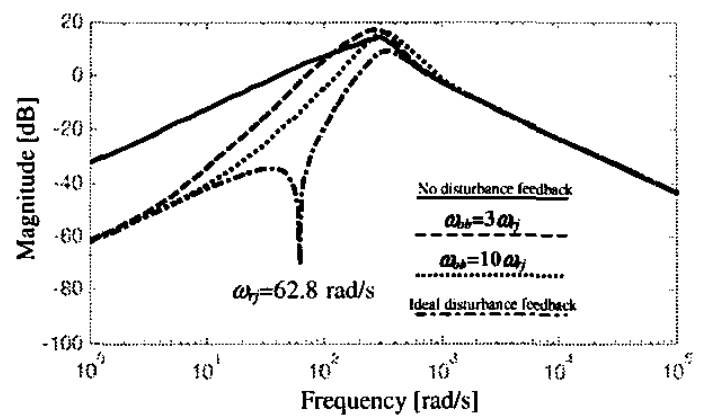

(a) Tuning assuming an ideal disturbance observer

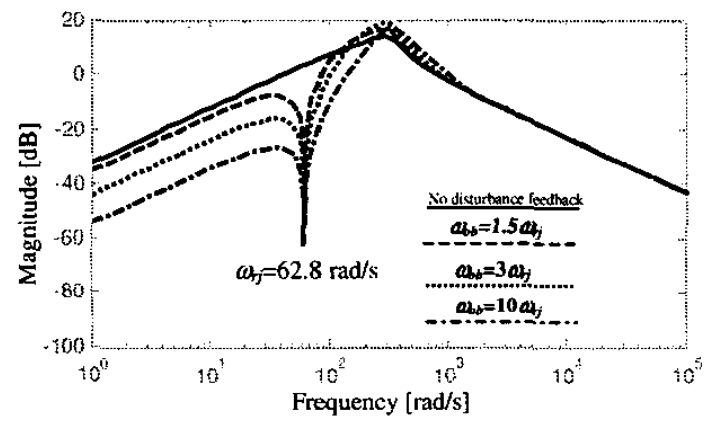

(b) Tuning assuming a non-ideal disturbance observer

Figure 6. Bode magnitude plots of the closed-loop regulation dynamics

Case (i) - assume an idea (high-bandwidth) disturbance observer. In this case, the disturbance torque feedback is assumed ideal and the effects of the observer are of sufficient bandwidth that they can be neglected. The disturbance feedback gains $K_{p d}$ and $K_{d d}$, are initially chosen according to (11) and (12) where $\omega_{r j}=62.8 \mathrm{rad} / \mathrm{s}$. To illustrate the influence of the observer on the resulting regulation performance, Fig. 6(a) compares the regulation bode magnitude plots $\left(\omega_{i} / t_{d}\right)$ as the observer bandwidth, $\omega_{b b}$ is reduced, (see (16) and (17)). Also shown is the regulation performance without additional disturbance feedback, i.e. in this case no disturbance feedback is implemented. It can be seen that the closed-loop dynamics induced by the presence of the observer impairs the ability of the closed-loop system to attenuate $\omega_{j j}$, even using a relatively high bandwidth observer. However, in general, the low frequency disturbance rejection performance is improved with the implementation of disturbance feedback.

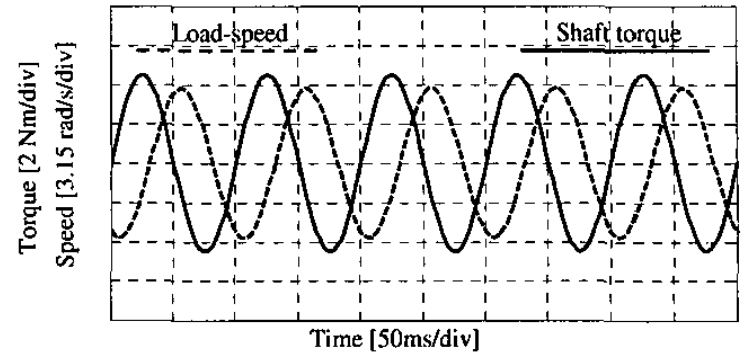

Figure 7. Simulated regulation responses with no disturbance feedback

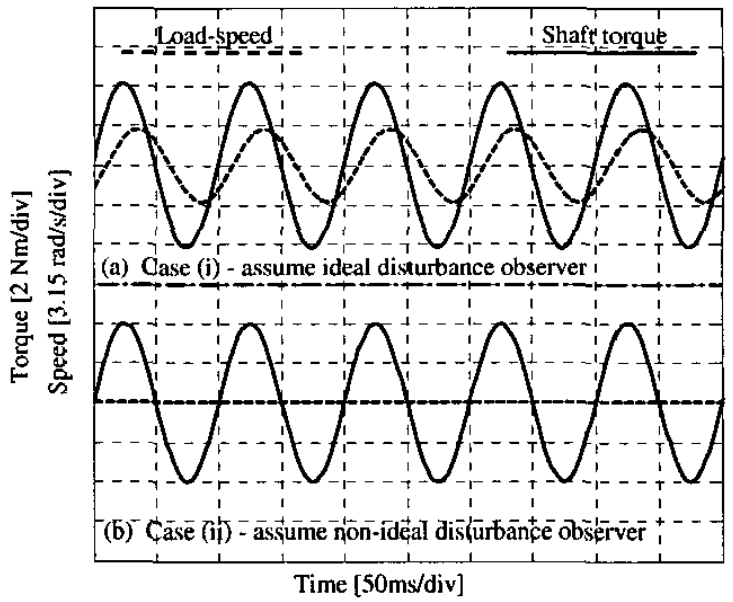

Figure 8. Simulated regulation responses with disturbance feedback $\omega_{b b}=3 \omega_{k j}$

Case (ii) - assume a non-ideal disturbance observer. The disturbance gains are now chosen according to (20-23) where $\omega_{j}=62.8 \mathrm{rad} /$, i.e. the disturbance torque feedback is assumed non-ideal and the dynamics of the disturbance observer are included in the tuning procedure. Figure 6(b) illustrates the magnitude plots as $\omega_{b b}$ is reduced. It can be seen that reducing the speed of the observer does not significantly influence the control objective, i.e. the attenuation at $\omega_{j}$ remains constant.

Figure 7 illustrates the time domain regulation performance in response to a sinusoidal load-side disturbance of $4 \mathrm{Nm}, 62.8 \mathrm{rad} / \mathrm{s}(10 \mathrm{~Hz})$, showing both the load-speed and the shaft-torque when no disturbance feedback is implemented. Figure 8(a) illustrates the response with disturbance feedback for case (i) and Fig. 8(b) illustrates the response for case (ii), where, for both cases, $\omega_{b b}=3 \omega_{j}$. It can be seen that significant improvement of the regulation performance is obtained in case (ii) where the load perturbations are completely rejected from the loadspeed, compared with case (i) where only a small attenuation of the load perturbations is evident. 


\section{EXPERIMENTAL RESULTS}

The proposed control techniques, simulation results $s_{c}$ and observations are now validated on an experimental testfacility as illustrated in Fig 9, comprising of $2 \times 2.2 \mathrm{~kW}$ brushless permanent magnet (PM) servo-machines mounted in a back-to-back configuration to provide a servo-drive and dynamic load, described by the parameters given in Table I. An integrated $20 \mathrm{Nm}$, SAW-based torque transducer forms the interconnecting shaft between the two servo-machines, Fig 9(a), and is used to realize the RRC and disturbance rejection control structures. The control algorithms, sensor inputs and control outputs are realized via a DSP-based, dSPACE system as illustrated schematically in Fig. 9(b).

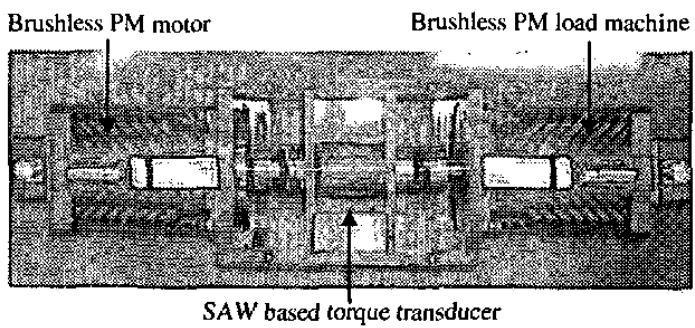

(a) Mechanical system overview

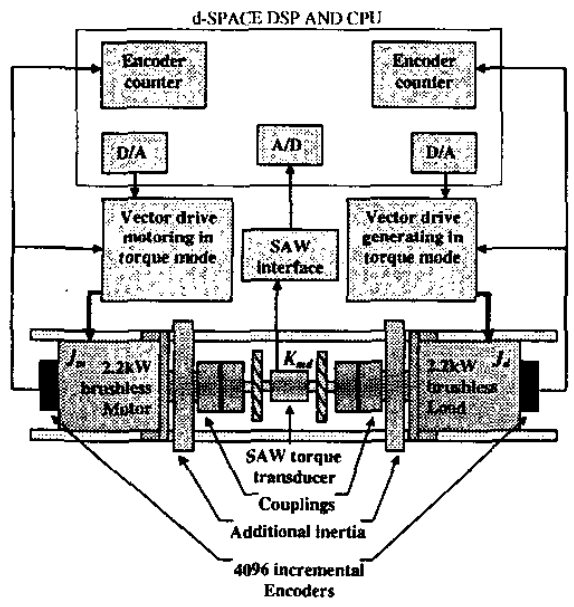

(b) Functions of components

Figure 9. Experimental facility, components and control system

\section{A. Tracking performance}

The experimental tracking results presented in Fig. 10 are in response to a $10 \mathrm{rad} / \mathrm{s}$ step reference demand, this therefore depicts the same events used in the simulation results of Fig. 5. Consequently, the extra flexibility afforded by RRC is shown to be sufficient to simultaneously impart optimal closed-loop load-side performance whilst also allowing the independent or virtual selection of inertia ratio.

\section{B. Regulation performance}

To evaluate the regulation performance, the load-side servo-machine generated a $4 \mathrm{Nm}$ sinusoidal disturbance torque with a frequency of $62.8 \mathrm{rad} / \mathrm{s}(10 \mathrm{~Hz})$. It was found that when disturbance feedback was implemented, a disturbance observer with a bandwidth $\omega_{b b}>50 \mathrm{~Hz}\left(5 \omega_{j}\right.$ in this case) caused controller instability due to the noise injected into the closed-loop system. Consequently, to provide valid experimental results, the observer bandwidth was limited to $\omega_{b b} \leq 3 \omega_{j}$.

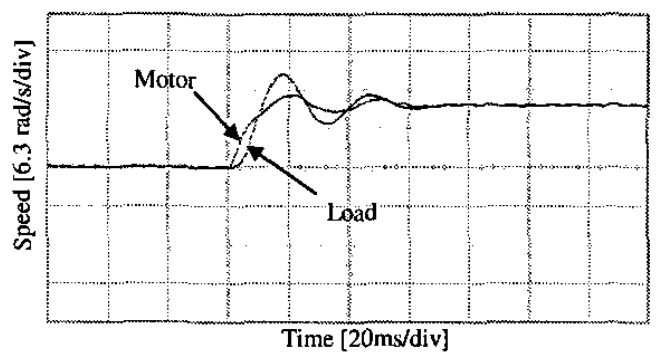

(a) PI controller with $\omega_{x}=0.88 \omega_{a}$

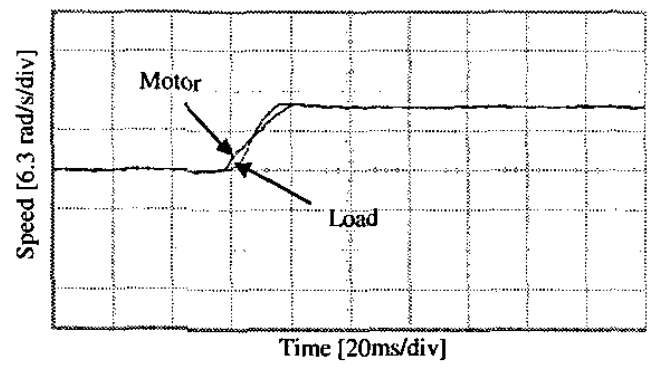

(b) RRC controller with $\omega_{x}=0.88 \omega_{x}$

Figure 10. Measured tracking step responses for the experimental twoinertia servo-drive system

Figure 11 illustrates the regulation response when no disturbance feedback is implemented within the controller structure showing both the load-speed and the shaft-torque. Fig. 12(a) illustrates the responses with disturbance feedback when the feedback gains are chosen assuming an ideal disturbance observer, case (i) and Fig. 12(b) assuming a non-ideal disturbance observer, case (ii), where for each case, $\omega_{b b}=3 \omega_{j}$. These results therefore depict the same events used in the simulation results of Figs. 7, 8(a) and 8(b).

To illustrate the effects of a further reduction in observer speed, the experimental results in Fig. 12(a) and 12(b) are repeated for $\omega_{b b}=1.5 \omega_{i j}$, i.e. the observer bandwidth is halved, the results being presented in Figs. 13(a) and 13(b). It can be seen by comparison of Figs. 12(a) and 13(a) that reducing the speed of the observer for case (i) significantly deteriorates the control objective i.e. the attenuation of the load-torque perturbations is further reduced and comparable with the results obtained with no disturbance feedback (Fig. 11), whilst comparing Figs. 12(b) and 13(b) for case (ii), it can be seen that the load perturbations are now rejected from 
the load-speed and the rejection performance is not unduly influenced by the speed of the observer.

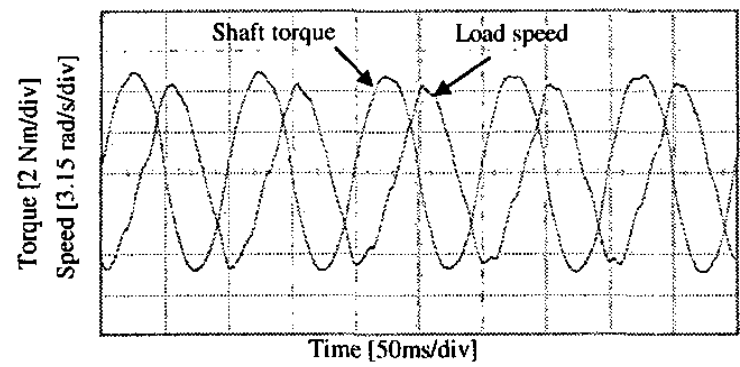

Figure 11. Experimental regulation responses with no disturbance feedback

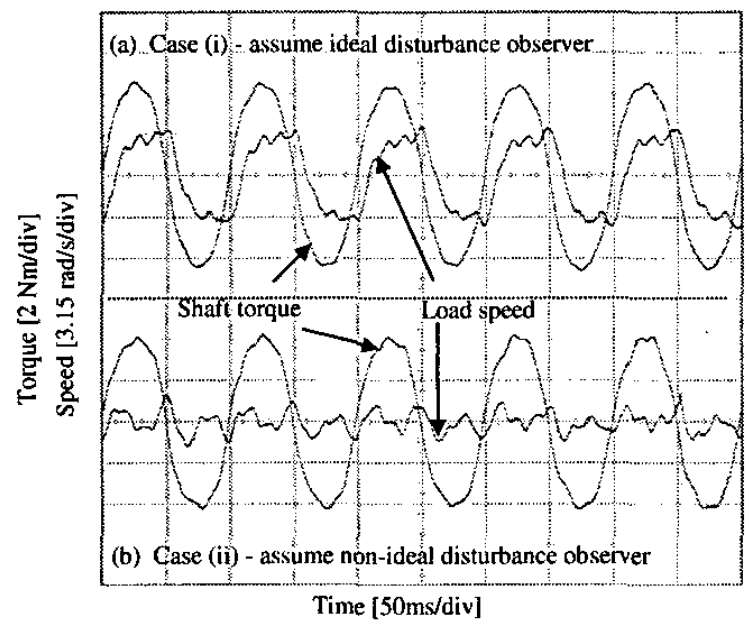

Figure 12. Experimental regulation responses with disturbance feedback $\omega_{t b}=3 \omega_{i j}$

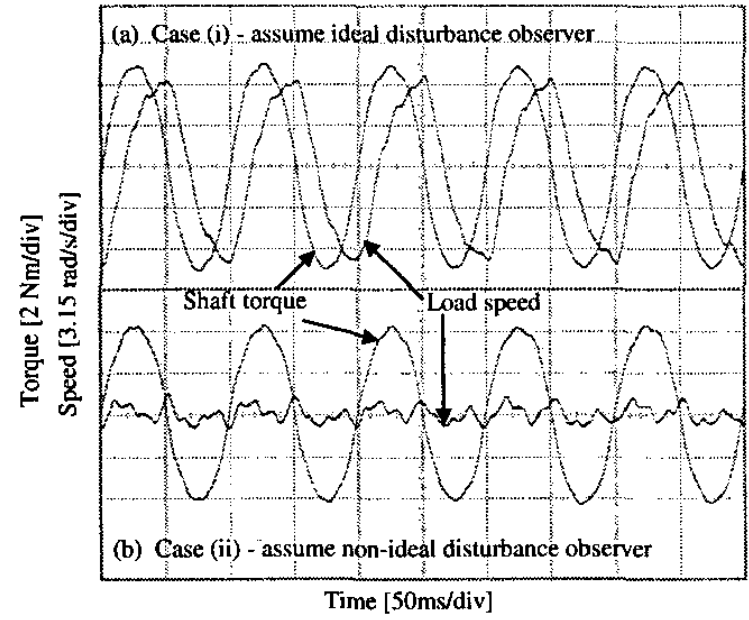

Figure 13. Experimental regulation responses with disturbance feedback. $\omega_{t b}=1.5 \omega_{r j}$

This is of significant practical importance for the system under investigation where sensor noise (torque transducer and encoder in this case) has a significant impact on the allowable speed of the observer.

\section{CONCLUSIONS}

A comparative study of compensation schemes for the control of multi-inertia mechanical systems has been reported. An improved technique based on the measurement of shaft torque using a SAW-based torque sensor is demonstrated to improve dynamic tracking performance, whilst additionally rejecting periodic load side disturbances. The proposed scheme allows assignment of the closed-loop zeros, thus enabling the rejection of a specific periodic load disturbance, and independent tuning of tracking and regulation dynamics.

\section{ACKNOWLEDGMENTS}

The authors acknowledge the UK Engineering and Physical Science Research Council (EPSRC), and Sensor Technology Ltd., Banbury, UK, for the provision of an EPSRC CASE studentship.

\section{REFERENCES}

[1] A. Lonsdale, "Dynamic rotary torque measurement using surface acoustic waves," Sensors, Vol. 18, no. 10, Oct. 2001, pp. 51-56.

[2] Y. Hori, H. Sawada and Y. Chun, "Slow resonance ratio control for vibration suppression and disturbance rejection in torsional system," IEEE trans. Ind. Elect., Vol. 46, No. 1, February 1999, pp. 162-8.

[3] K. Yuki, T. Murakami, K. Ohnishi, "Vibration control of 2-mass resonant system by resonance ratio control," Industrial Electronics, Control, and Instrumentation. Proceedings of the IECON '93., Intemational Conference on, 15-19 Nov 1993 Vol. 3, pp. 2009-14.

[4] S. Mortimoto, A. Hamamoto, Y. Takeda, "Vibration control of twomass system with low inertia ratio considering practical use," Electrical Engineering in Japan, Vol. 125, No. 2, 1998, pp. 1-9.

[5] Th. Euteback, J.M. Pacas, "Damping of torsional vibrations in highdynamic-drives," 8th European conference on power electronics and applications. EPE'99. EPE Assoc., Brussels, Belgium, CD-ROM pp. $10,1999$.

[6] M. Menne and O. Bitsche, " Comparison of drivetrain oscillation damping-algorithms for electric vehicles," EVS 18 Berlin, 2001.

[7] Solbodan N. Vukosavic and Milic R. Stojic, "Suppression of torsional oscillations in a high performance speed servo drive," IEEE Trans. Ind. Elect., Vol. 45, No. 1, February 1998.

[8] T.M. O'Sullivan, N. Schofield and C.M. Bingham, "Simulation and experimental validation of induction machines dynamics driving multi-inertia loads," Joumal of Applied Electromagnetics and Mechanics (JAEM), Vol. 19, (2003).

[9] Gene Franklin, J. David Powell and Abbas Emami-Naeini "Feedback Control of Dynamic Systems", book, Prentice-Hall, ISBN: 0-13032393-4, 2002 